\title{
Prevalence of Benign Vocal Fold Lesions in Ear, Nose, and Throat Outpatient Unit of Dr. Soetomo General Hospital, Surabaya, Indonesia
}

\author{
Lucia Miranti Hardianingwati ${ }^{1}$, Diar Mia Ardani2 ${ }^{*}$ \\ ${ }^{1}$ Department of Otorhinolaryngology - Head and Neck Surgery, Faculty of Medicine, Universitas Airlangga - Dr. Soetomo \\ General Hospital Surabaya, Indonesia \\ ${ }^{2}$ Division of Pharyngeal Larynx, Department of Otorhinolaryngology - Head and Neck Surgerye, Faculty of Medicine, \\ Universitas Airlangga - Dr. Soetomo General Hospital Surabaya, Indonesia
}

\section{A R T I C L E I N F O}

\section{Article history:}

Received 12 May 2020

Received in revised form 06 June

2020

Accepted 08 June 2020

Available online 30 June 2020

Keywords:

Nodule,

Polyp,

Vocal fold,

Dysphonia.

*) Corresponding author:

bhandokodaeng@yahoo.com

\begin{abstract}
A B S T R A C T
Introduction: Benign vocal fold lesions reduce the efficiency of sound production. Reports of dysphonia cases caused by vocal principles in Indonesia are still very limited. This study aimed to determine incidence and prevalence of benign vocal fold lesions, namely vocal cord nodules, cysts, and polyps.

Methods: A descriptive retrospective study was conducted using patient's medical record of Ear, Nose, and Throat (ENT) Outpatient Unit. Dysphonia patients with benign vocal cord abnormalities were identified. The data analyzed using descriptive analytic.

Results: There were 20 patients with benign vocal fold lesions, consisting of 13 patients $(65 \%)$ with nodules, 3 patients (15\%) with polyps, and 4 patients $(20 \%)$ with cysts. The ratio of male and female patients was 1: 1 . Most patients belonged to age group of 20-59 years (12 patients; $60 \%$ ). In term of occupation, most patients belonged to group III, which is a group of workers who are not professional voice users (12 patients; 60\%). Most vocal fold lesions were found in the $1 / 3$ of bilateral anterior (17 patients; $85 \%)$. Most therapy was non-operative in 13 patients $(65 \%)$. Conclusion: Benign vocal fold lesions, including vocal cord nodules, polyps, and cysts, are found in all patients with dysphonic complaints. The prevalence of dysphonia symptoms is quite high every year, but only a small portion are diagnosed with benign vocal cord lesion.
\end{abstract}

\section{Introduction}

Benign vocal fold lesions are abnormal masses in laryngeal tissue that are overgrown and uncoordinated. ${ }^{1}$ Benign vocal fold lesions reduce efficiency of sound production that leads to hoarse and breathy voice, so the patient needs more effort to talk. Thus, the patient complains of fatigue and discomfort in the neck and throat. Patients with benign vocal fold lesions do not feel pain. The uneven surface of the vocal cords causes mucus to often get lodged, causing a lump in throat and coughing reactions. ${ }^{2}$

Benign vocal fold lesions can be divided into two, namely neoplastic and non-neoplastic. Non-neoplastic vocal fold lesions include vocal cord nodules, vocal cord polyps and vocal cord cysts. ${ }^{3}$ The extroverted personality who talks a lot and occupations with high voice requirements becomes a risk factor for benign vocal fold lesions. Other risk factors are smoking, stomach acid reflux, allergies and infections. ${ }^{4}$

Benign vocal fold lesions are common cases, but its prevalence is difficult to determine. In 2011, Cohen et al. conducted a retrospective study of the prevalence and causes of dysphonia in the United States. The study compared differences in the etiology of dysphonia determined by primary care physicians and ENT specialists. The research data were taken from dysphonia code according to the International Classification of Disease (ICD) code 9 recorded in the United States in the period of January 2004 to December 2008. The total population obtained in that period was fifty-five million patients. The prevalence of patients with dysphonia diagnosis from the total population was $1 \%$, and approximately $11 \%$ of patients with dysphonia were caused by benign vocal fold lesions. Benign vocal 
fold lesions consist of vocal cord polyps, vocal cord nodules, vocal cord abscesses, vocal cords cellulitis, vocal cords granulomas, and vocal cords leukoplakia. ${ }^{4,5}$

There has been no report regarding incidence and prevalence of benign vocal fold lesions in Dr. Soetomo General Academic Hospital, Surabaya, Indonesia. This study aimed to determine the incidence and prevalence of benign vocal fold lesions in the Ear, Nose, and Throat (ENT) Outpatient Unit of Dr. Soetomo General Hospital, Surabaya, Indonesia.

\section{Methods}

A retrospective descriptive study was conducted at the ENT Outpatient Unit of Dr. Soetomo General Academic Hospital, Surabaya, Indonesia. Data collected in this study were 20 patients in the ENT Outpatient from June 2015 to June 2016.

The accessible population was patients with dysphonia complaints who visited ENT Clinic. The study sample was dysphonia patients with direct rigid and flexible laryngoscopy examination that showed benign vocal fold lesions, including vocal cord nodules, vocal cords cysts, or vocal cord occupation. The inclusion criteria included patients with symptoms of dysphonia, patients with a diagnosis of benign vocal fold lesions (nodules, cysts, or vocal cord polyps), and complete patient data. The patient's data included patient's identity, history, examination results, and diagnosis after direct rigid or flexible laryngoscopy procedures. The exclusion criteria were history of malignancy with stage III $\leq$ and incomplete medical record. The collected data were taken from medical record and arranged in a table based on age, sex, occupation, types of benign vocal fold lesions, location of lesions and the therapy given.

The type of occupation in this study was divided into four groups according to the level of voice used during work based on Koufmn and Isaacson's classification. Level I refers to elite vocal performers, namely singers and radio broadcasters. Level II is a professional voice user such as a lecturer. Level III is non-vocal professionals, including teachers, traders, and students. Level IV is non-vocal non-professional, such as housewives and farmers [6]. Measurement data were analyzed and presented in the form of frequency distributions

\section{Results}

The results of medical record data obtained 475 patients with dysphonia from June 2015 to June 2016. Of all 475 patients, there were 49 patients with advanced malignancy and 78 incomplete medical record. There were 23 patients diagnosed with benign vocal cord lesion and the rest had other cases. There were 20 patients with complete medical records and benign vocal cord lesions.

There were 10 male patients $(50 \%)$ and 10 female patients $(50 \%)$. The patient's age ranged from 5-73 years old, with a mean of 33.55 years. The age group with the highest number of patients ranged from 20-59 years old (12 patients; $60 \%$ ). The distribution of occupational groups according to Koufmn and Isaacson was shown in Table 1.

\section{Types of Benign Vocal Fold Lesions}

The distribution of types of benign vocal fold lesions was shown in Table 1. In this study, the most common type of vocal fold lesions was vocal cord nodules (13 cases; $65 \%)$. The least type of lesion was vocal cord polyps ( 3 cases; $15.00 \%$ ).

The incidence of vocal cord nodules in this study was the highest when compared to other types of benign vocal fold lesions $(2.73 \%)$, followed by vocal cord polyps $(0.63 \%)$ and vocal cord cysts $(0.84 \%)$. The prevalence of vocal cord nodules in this study was $4.42 \%$, while the prevalence of vocal cord polyps and vocal cord cysts was $1.26 \%$ and $2.31 \%$, respectively.

Table 1. Distribution of Subject Characteristics

\begin{tabular}{lc}
\hline \multicolumn{1}{c}{ Characteristic } & Frequency (\%) \\
\hline Sex & $10(50.00)$ \\
\hline Male & $10(50.00)$ \\
\hline Age (year) & $4(20.00)$ \\
\hline $1-9$ & $2(10.00)$ \\
\hline $10-19$ & $12(60.00)$ \\
\hline $20-59$ & $2(10.00)$ \\
\hline$\geq 60$ & $3(15.00)$ \\
\hline Occupation* & $1(5.00)$ \\
\hline I & $12(60.00)$ \\
\hline II & $4(20.00)$ \\
\hline III & \\
\hline IV & $13(65.00)$ \\
\hline Benign Vocal Fold Lesions & $3(15.00)$ \\
\hline Nodule & $4(20.00)$ \\
\hline Polyp & 11 \\
\hline Cyst & \\
\hline
\end{tabular}

*Group I = elite vocal performer, II = professional voice user, III = non-vocal professional, IV = non-vocal non-professional.

\section{Location of Vocal Fold Lesions}

Benign vocal fold lesions were grouped according to the location of lesion, namely $1 / 3$ of anterior vocal cord, $1 / 3$ of medial vocal cord, unilateral, and bilateral. The location of most vocal cord polyps was in the $1 / 3$ of anterior unilateral vocal cord (2 patients; 66.7\%), while the least was in the $1 / 3$ of medial unilateral vocal cord (1 patient; 33.3\%). Most vocal cord cysts were in the $1 / 3$ of anterior unilateral vocal cord ( 3 patients; $75 \%$ ), and the least was in the $1 / 3$ of medial unilateral vocal cord (1 patient; $25 \%$ ). The most vocal cord nodules were in the $1 / 3$ of anterior bilateral vocal cord (11 patients; $91.7 \%$ ), and the least was located in the $1 / 3$ of anti-lateral unilateral vocal cord (1 patient).

The distribution of group of benign vocal fold lesions was shown in Table 2. 


\section{Therapy and Results}

Patients with benign vocal fold lesions were grouped according to the type of therapy given. The most therapeutic group was non-operative (13 patients; 65\%), and the least was non-control group (2 patients; $10 \%$ ), as shown in Table 6. The no-show non-operative patients were as many as 9 patients $(69.2 \%)$, and operative patients who felt improvement were 3 patients $(60 \%$; Table 3 ).

Table 2. Distribution of Location of Benign Vocal Fold Lesions

\begin{tabular}{ccccccc}
\hline \multirow{2}{*}{ Vocal Fold Location } & \multicolumn{2}{c}{ Polyp } & \multicolumn{2}{c}{ Cyst } & \multicolumn{2}{c}{ Nodule (\%) } \\
& Unilateral & Bilateral & Unilateral & Bilateral & Unilateral & Bilateral \\
\hline $1 / 3$ Anterior & $2(66.70)$ & - & $3(75.00)$ & - & $1(100.00)$ & $11(91.70)$ \\
\hline $1 / 3$ Medial & $1(33.30)$ & - & $1(25.00)$ & - & - & $1(8.30)$ \\
\hline
\end{tabular}

Table 3. Types of Therapy and Results of Therapy in Patients with Benign Vocal Fold Lesions

\begin{tabular}{cccccccccc}
\hline \multirow{2}{*}{ Therapy } & \multicolumn{3}{c}{ Polyp } & \multicolumn{3}{c}{ Cyst } & & \multicolumn{3}{c}{ Nodule (\%) } \\
\cline { 2 - 11 } & Improved & Stable & No-show & Improved & Stable & No-show & Improved & Stable & No-show \\
\hline Non-operative & - & - & $1(33.30)$ & - & - & - & $4(33.30)$ & - & $8(66.70)$ \\
\hline Operative & - & - & - & $3(75.00)$ & $1(25.00)$ & - & - & $1(100.00)$ & - \\
\hline No-show & - & - & $2(66.70)$ & - & - & - & - & - & - \\
\hline
\end{tabular}

\section{Discussion}

Direct rigid and flexible laryngoscopy can detect abnormalities in larynx such as inflammation, lesions and narrowing of airway. Direct laryngoscope can also be used to perform biopsy of laryngeal tissue. ${ }^{7}$ Benign vocal fold lesions are less common than malignant disorders. These abnormalities are divided into two, namely non-neoplastic and neoplastic tumors. Nonneoplastic vocal fold lesions occur due to infection, trauma, and degeneration. Some examples of nonneoplastic lesions are vocal cord nodules, vocal cord polyps, and vocal cord cysts. Vocal cord nodules are always bilateral and almost symmetrical. On stroboscopic examination, vocal cord nodules will appear in a decreased mucosal wave. The nodules will shrink or disappear with sound therapy. Vocal cord polyps can occur unilateral or bilateral with clear or reddish exophytic lesions in hemorrhagic polyps. The size of vocal cord polyps does not change with sound therapy. Vocal cord cysts can be unilateral or bilateral. It is located near ligaments or in the subepithelial cavity. The subepithelial cavity is the area just below the vocal cord epithelium. . $^{3,4,8}$

In this study, the ratio of male and female patients with benign vocal fold lesions was 1: 1 , thus indicates that both genders have similar risk of vocal cord disorders. Benign vocal fold lesions are mostly found in female because female more often use sounds excessively. ${ }^{8}$ In the pre-menstrual period, female experience premenstrual vocal syndrome (PMVS), which is a change in vocal cords' stability due to hormonal fluctuations. PMVS is characterized by being unable to reach high notes and losing voice power. During pre-menstrual period, there is also dryness of larynx due to unbalanced estrogen and progesterone levels. Dry larynx triggers the patient to clear throat that often leads to vocal cord nodules. ${ }^{9}$

Other studies also found similar findings, as benign vocal fold lesions were mostly found in this age range. ${ }^{10,11}$ This result indicated that vocal fold lesions occur in patients in the age group of workers. Patients within working age who frequently use sounds will have a greater risk for suffering from benign vocal fold disorders. ${ }^{11,12}$

The Voice Handicap Index (VHI) score is a questionnaire containing 30 questions about the quality of life of dysphonia patients with good validity and reliability. The total VHI score is between 0 (without disability) to 120 (maximum disability). The VHI provides information about patients' perceptions of the level of sound defects in daily life. Previous retrospective studies have shown that level of sound requirements associated with lifestyle and work influences VHI scores. The highest number of working age patients in this study showed that working age patients highly need to speak. This also affected the VHI score. A high VHI score encourages working age patients to come for treatment. ${ }^{13}$

Type of occupation has a close relationship with benign vocal cord lesions. The type of occupation that requires loud noises with high frequency is a risk factor for benign vocal folds. Koufman and Isaacson classified voice into four groups. In this study, most patients belonged to group III. The type of occupation included in group III often involve vocal abuse because this group works using sound as the main need without professional training. ${ }^{6}$ There are several types of occupation in group III that often require moderate sound quality but high sound load. ${ }^{14}$ The intended sound load is long working hours, noisy environment, and inadequate work facilities. The excessive number of students in each classroom also influences the sound burden on the type of work as a teacher. ${ }^{15}$

The most common type of benign vocal fold lesions in this study was vocal cord nodules. This result is consistent with other studies. ${ }^{11}$ Most patients with vocal cord nodules have jobs with voice as their primary need. This causes patients with vocal cord nodules to 
attend treatment more quickly than other benign vocal fold lesions. ${ }^{4}$

The results of previous studies were similar to the current study which showed that $47 \%$ of patients had vocal cord abnormalities in $1 / 3$ anterior, $11 \%$ in $1 / 3$ medial and $22 \%$ in $1 / 3$ posterior vocal cords. ${ }^{10,11}$ The $1 / 3$ anterior of vocal cords are part of the membrane. When vocal cords vibrate, the membrane experiences friction and collision between the greatest vocal cords. Longterm and strong vibrations causing vascular congestion accompanied by swelling in vocal cord membrane. This reason why benign vocal fold abnormality is commonly found in the $1 / 3$ anterior part of vocal cord. ${ }^{16}$ In this study, one patient experienced unilateral vocal cord nodules, as caused by several possibilities. The patient's vocal cord nodules were detected so early that the possibility of reactive lesions in the contralateral side vocal cord has not yet formed. Facilities for vocal cord examination at the study site were direct rigid and flexible laryngoscopy. Direct rigid and flexible need patient cooperation and operator skills. Unilateral vocal fold lesions can be detected by tele-laryngoscopy (stroboscopy) as much as $79.8 \%$, while direct rigid laryngoscopy with general anesthesia can only diagnose of $60.7 \% .^{17}$

The non-operative therapy is an attempt to optimize the condition of larynx. The condition of larynx will be optimal with sound therapy that eliminates habits that can injure vocal cords such as screaming or whispering, using sounds in moderation and optimal hydration. Other health problems related to vocal cord irritation are also treated, such as gastric acid reflux and controlling allergies. Benign vocal fold lesions provide a very good response to non-operative therapy, hence it becomes primary choice. ${ }^{3,6,16}$

The operative therapy is more aimed at benign vocal cord polyps and vocal cord cysts. Benign vocal fold lesions that are exophytic, such as vocal cord polyps, and lesions that cause severe mucosal stiffness, such as ligamentous cysts, give poor results in non-operative therapy. Operative therapy is the first choice if the patient has dysphagia associated with aspiration, has a risk of airway obstruction, and a suspicion of malignancy. In patients who have undergone non-operative therapy but do not obtain results in accordance with patient's expectations, operative therapy can be used as an option. Operative therapy is also chosen if the need for sound for daily life is very important for the patient. ${ }^{3,6}$

Doloi and Khanna (2011) conducted a study of therapy given to patients with benign vocal fold disorders. Nonoperative therapy in the study included antibiotics, antiinflammatory, vapor inhalation, and voice rest. Meanwhile, operative therapy included excision with direct rectal laryngoscopy, excision with endoscopy, and external excision. ${ }^{18}$ The percentage of patients treated non-operatively in our study was quite high and showed good results. These results differ from a study conducted by Singhal et al., (2009) as they found that only $6 \%$ of patients treated non-operatively had good results. ${ }^{1}$ The results of Doloi and Khanna's study were likely affected by early detection of patient's vocal fold abnormalities. ${ }^{18}$

The limitation of this study relies on the method of diagnosing begin vocal fold lesions which still does not use stroboscopy. It is expected that the hospital provides the tool for better accuracy.

\section{Conclusion}

The number of patients with symptoms of dysphonia was enormous yet patients diagnosed with benign vocal cord lesion were very few. Reporting the number of cases of benign vocal cord lesion with dysphonia was used as data for further (specifically the data on the number of patients with benign vocal cord abnormalities).

\section{Conflict of Interest}

Lucia Miranti Hardianingwati and Diar Mia Ardani declare that they have no conflict of interest.

\section{References}

1. Singhal, P., et al., Benign tumors of the larynx: a clinical study of 50 cases. Indian journal of otolaryngology and head and neck surgery: official publication of the Association of Otolaryngologists of India, 2009. 61(Suppl 1): p. 26-30.

2. Martins, R.H.G., et al., Voice Disorders: Etiology and Diagnosis. Journal of Voice, 2016. 30(6): p. 761.e1-761.e9.

3. Phaniendra Kumar, V., et al., Phonomicrosurgery for benign vocal Fold lesions - our experience. Indian journal of otolaryngology and head and neck surgery : official publication of the Association of Otolaryngologists of India, 2003. 55(3): p. 184-186.

4. Sataloff, R.T., Professional Voice: The Science and Art of Clinical Care, 3-Volume Set. 2017: Plural Publishing.

5. Cohen, S.M., et al., Prevalence and causes of dysphonia in a large treatment-seeking population. The Laryngoscope, 2012. 122(2): p. 343-348.

6. Timing, Planning, and Decision Making in Phonosurgery, in Operative Techniques in Laryngology. 2008, Springer Berlin Heidelberg: Berlin, Heidelberg. p. 49-52.

7. Pott, L.M. and W.B. Murray, Review of video laryngoscopy and rigid fiberoptic laryngoscopy. Current Opinion in Anesthesiology, 2008. 21(6): p. 750-758.

8. Sharma, D.K., et al., Clinico-pathological study of 50 cases of tumours of larynx. Indian journal of otolaryngology and head and neck surgery : official publication of the Association of Otolaryngologists of India, 2013. 65(Suppl 1): p. 29-35.

9. Khare, V., The Influence of Sex Hormones on the Female Singing Voice: A Review of the Literature, 1971-2016. 2016.

10. Wani, A., et al., Benign mucosal fold lesion as a cause of hoarseness of voice-A clinical study. Otolaryngology, 2012. 2(3): p. 1-3.

11. Niebudek-Bogusz, E. and M. Śliwińska-Kowalska, An overview of occupational voice disorders in Poland. International journal of occupational medicine and environmental health, 2013. 26(5): p. 659669.

12. Bermingham, S.L., et al., The cost of somatisation among the workingage population in England for the year 2008-2009. Mental Health in Family Medicine, 2010. 7(2): p. 71.

13. Behrman, A., L. Sulica, and T. He, Factors Predicting Patient Perception of Dysphonia Caused by Benign Vocal Fold Lesions. The Laryngoscope, 2004. 114(10): p. 1693-1700.

14. Boltežar, L. and M. Šereg Bahar, Voice Disorders in Occupations with Vocal Load in Slovenia. Zdravstveno varstvo, 2014. 53(4): p. 304-310.

15. Moy, F.M., et al., Determinants and Effects of Voice Disorders among Secondary School Teachers in Peninsular Malaysia Using a Validated Malay Version of VHI-10. PLOS ONE, 2015. 10(11): p. e0141963.

16. Wang, C.T., et al., Intralesional steroid injection for benign vocal fold disorders: a systematic review and meta-analysis. The Laryngoscope, 2013. 123(1): p. 197-203.

17. Neto, J.A.M., et al., Comparison between telelaryngoscopy and suspension laryngoscopy in the diagnosis of benign vocal fold lesions. Brazilian journal of otorhinolaryngology, 2008. 74(6): p. 869-875.

18. Doloi, P.K. and S. Khanna, A study of management of benign lesion of the larynx. International Journal of Phonosurgery and Laryngology, 2011. July-December(2): p. 61-64. 